\title{
Intergroup Rhabdomyosarcoma Group Ib
}

National Cancer Institute

\section{Source}

National Cancer Institute. Intergroup Rhabdomyosarcoma Group Ib. NCI Thesaurus.

Code C148014.

Infiltrative, beyond site of origin; negative lymph nodes. 